\title{
Segmentação Automática de Imagens Térmicas das Mamas Utilizando Limiarização com Refinamento Adaptativo
}

\author{
Matheus F. O. Baffa ${ }^{1}$, Deivison J. M. Cheloni ${ }^{1}$, Lucas G. Lattari ${ }^{1}$ \\ ${ }^{1}$ Departamento Acadêmico da Ciência da Computação - Instituto Federal de Educação, \\ Ciência e Tecnologia do Sudeste de Minas Gerais (IFSEMG) \\ \{mfreitas826, deivison.junior\}@gmail.com, lucas.lattari@ifsudestemg.edu.br
}

\begin{abstract}
Breast cancer is the most common type of cancer in the world. New cases of cancer are detected every year, and $25 \%$ of them are breast cancer. It is known that early diagnosis is critical for the prognosis of patients, and image based techniques has been developed to guide an effective, minimally invasive and cheaper way to diagnose breast cancer. In this work, we propose an automatic segmentation method based on adaptive thresholding. The experimental results show that our method is competitive with other works on both solution quality and runtime performance, achieving $96 \%$ of accuracy and $98 \%$ of sensibility. Furthermore, it is simpler to implement, computationally efficient and suitable for real time applications.

Resumo. O câncer de mama é o tipo mais comum de câncer no mundo. Todo ano são detectados milhares de casos de câncer, e destes, 25\% são de mama. Sabendo que o diagnóstico precoce é crítico para o prognóstico do paciente, novas tecnologias à base de análise de imagens são desenvolvidas para guiar um diagnóstico eficaz e menos invasivo. Nesse artigo, é desenvolvido um novo método de segmentação de imagens das mamas em imagens termográficas utilizando limiarização com refinamento adaptativo. Esse método se mostrou eficaz com aproximadamente $96 \%$ de acurácia e $98 \%$ de sensibilidade. Além disso, a abordagem proposta é simples de ser implementada computacionalmente, é eficiente e apropriada para aplicações em tempo real.
\end{abstract}

\section{Introdução}

Para o ano de 2015, o Instituto Nacional do Câncer (INCA) previu um total de 500 mil novos casos de câncer somente no Brasil. Dos novos casos, estima-se que $25 \%$ seriam de câncer de mama. Essas doenças afetam homens e mulheres das mais diversas idades, sendo mais frequente em mulheres acima dos 50 anos. De acordo com uma pesquisa divulgada pelo INCA, estima-se que o câncer de mama foi responsável pela morte de aproximadamente 14 mil mulheres no ano de 2013 [INCA 2015].

A detecção precoce do câncer pode aumentar as chances de cura do paciente. Em média, os pacientes diagnosticados no início do desenvolvimento do tumor podem ter até $95 \%$ de chance de cura [HCB 2015]. O INCA divulga que não há apenas uma causa para a doença, mas diversos fatores que desencadeiam o câncer, como fatores genéticos, hormonais e ambientais.

Atualmente, existem diversas formas de se diagnosticar o câncer de mama. Um exemplo a ser considerado é o autoexame, em que a própria pessoa verifica se há alguma anomalia. Outra forma é através de exames de imagens como a mamografia, o 
ultrassom e a ressonância magnética, em que através da análise de imagens, o médico irá verificar a existência de tumores. Além destas, as imagens termográficas aplicadas a saúde têm se mostrado promissoras, sendo utilizadas por vários pesquisadores para se detectar anomalias nas mamas [Arora et al. 2008, Motta 2010]. Segundo Gore e Xu [Gore and Xu 2014], o corpo humano possui alta simetria em relação a sua distribuição térmica, que se mantém estável mesmo após longos períodos de observação. Qualquer alteração na simetria térmica pode indicar a existência de doenças.

Para se diagnosticar câncer em imagens termográficas, torna-se necessário identificar a região de interesse (ROI) que compõe as mamas, removendo o fundo da cena que não é desejado para a análise. Somente a ROI é avaliada posteriormente para a identificação de doenças.

O trabalho de [Motta 2010] realiza uma segmentação automática das mamas em imagens térmicas. Seu algoritmo, representado na Figura 1, realiza a segmentação utilizando morfologia matemática, detecção de contornos usando filtros de Canny, detecção de formas através da transformada de Hough e limiarização adaptativa. A acurácia média da avaliação quantitativa foi de aproximadamente $96 \%$ em relação ao ground truth.

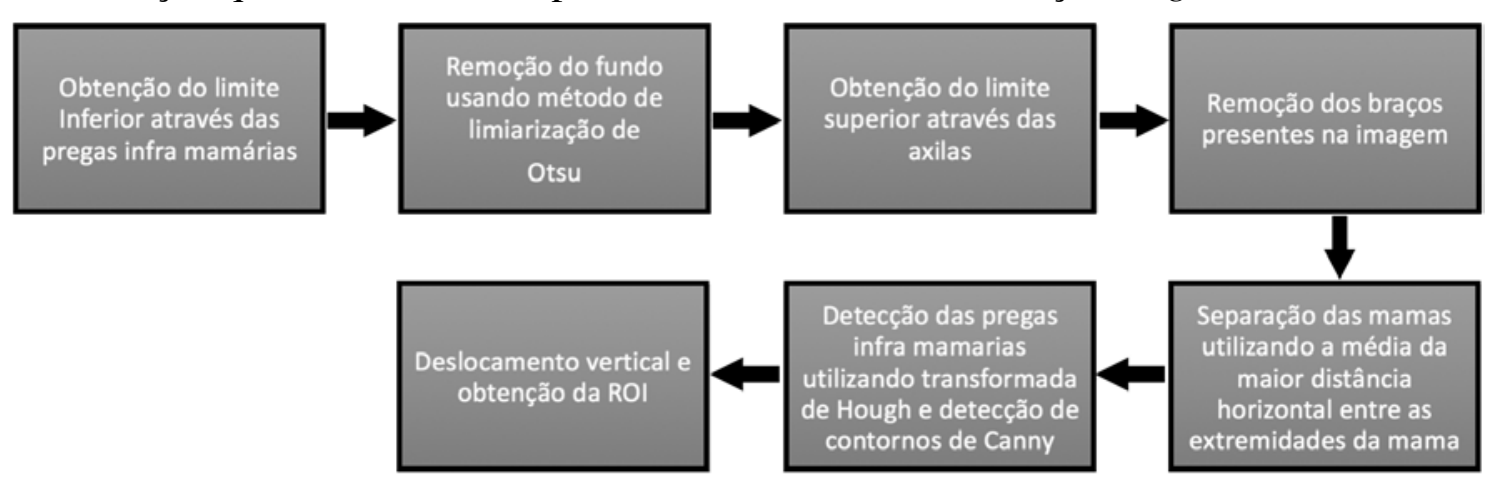

Figura 1. Fluxograma do método de segmentação proposto em [Motta 2010].

O trabalho de [Marques 2012] também apresenta uma abordagem diferente à segmentação automática das mamas. Seu método, descrito na Figura 2, inclui detecção de contornos, limiarização, crescimento de regiões, ajuste de curvas por mínimo quadrado e B-Splines.

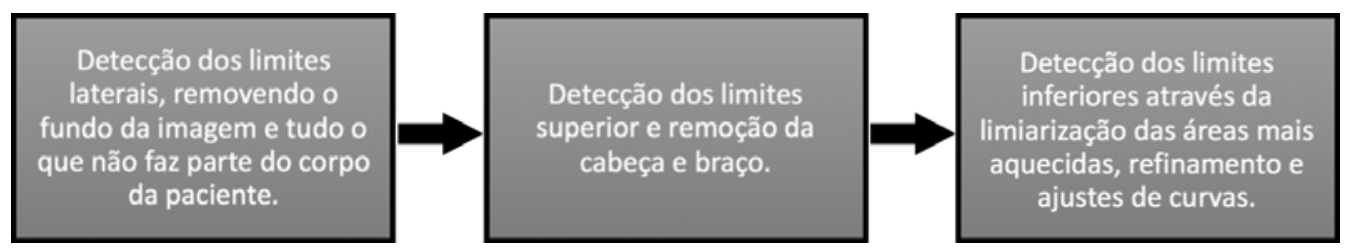

Figura 2. Fluxograma do método de segmentação proposto em [Marques 2012].

O método de [Marques 2012] apresenta uma acurácia de 97\%. As imagens da ROI (Region of Interest) segmentadas são comparadas com um ground truth gerado a partir de um aplicativo usando tablets para a segmentação manual das imagens.

Nesse artigo, é desenvolvido um novo método para a segmentação da região das mamas em imagens termográficas, usando o conceito de limiarização com refinamento adaptativo. Os resultados desse método se mostraram muito promissores, obtendo taxas médias de $96 \%$ de acurácia e $98 \%$ de sensibilidade. Algumas contribuições do presente trabalho são: 
- A criação de uma nova técnica de limiarização com refinamento adaptativo, que a partir da simetria térmica da imagem, identifica a região que potencialmente representa as pregas inframamárias, fundamental para a identificação das mamas. Essa descrição se mostra de alta qualidade, pois apresenta resultados competitivos e superiores a outros trabalhos da literatura, mesmo após a avaliação de resultados experimentais com centenas de imagens de teste obtidas em bases de dados científicas.

- A descrição de uma aplicação computacional que é mais simples de se implementar e portar em diversas arquiteturas computacionais, mesmo em dispositivos que dispõem de pouca memória. Além disso, o tempo de execução médio permite a possibilidade de usar o método proposto para aplicações em tempo real, como vídeos.

\section{Método Proposto}

As imagens termográficas das mamas são introduzidas às etapas do método proposto que é apresentado na Figura 3.

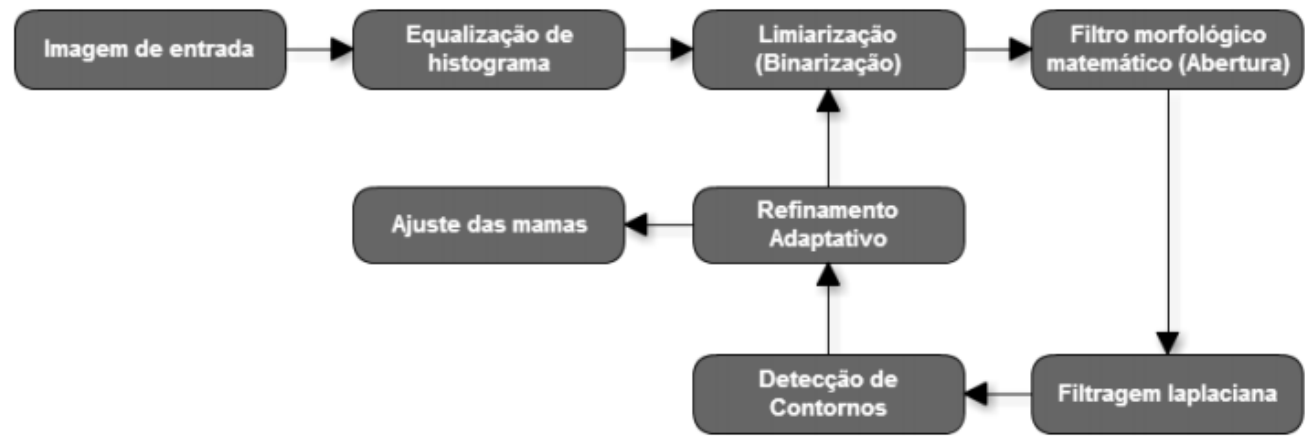

Figura 3. Fluxograma do método proposto neste trabalho.

\subsection{Limiarização por Refinamento Adaptativo}

Como mencionado em Marques [Marques 2012], as pregas inframamárias são indicadores dos limites inferiores das mamas. Além disso, esta região possui maior temperatura devido a sobreposição entre a parte inferior da mama e a parede torácica. Esta característica facilita a identificação computacional da prega inframamária e, posteriormente, das mamas.

Dessa forma, utilizando a limiarização com limiar $L$ elevado, torna-se possível realizar a segmentação das pregas inframamárias. Entretanto, experimentalmente, foi observado que o valor de $L$ ideal para a correta segmentação das pregas inframamárias é variável, dependendo do conteúdo da imagem térmica $I$. Para o cálculo desse valor ideal, este trabalho propõe uma abordagem de refinamento adaptativo, que tem por objetivo calcular o melhor valor de $L$ para qualquer $I$ considerado.

No início do método, $I$ é limiarizado com limiar elevado $(L=254)$. Após essa etapa, a imagem térmica é filtrada usando um operador morfológico de abertura. O objetivo da aplicação desse filtro é remover pequenos artefatos de uma imagem que deterioram o resultado final. 
Na próxima etapa, é realizada a filtragem laplaciana de $I$, após a limiarização utilizada para a obtenção da prega inframamária. Isso é feito para a obtenção dos contornos das pregas e, consequentemente, permitir uma análise geométrica para a identificação da região mamária.

A partir dos contornos obtidos, a área $A_{i}$ de cada segmento conexo $i \in I$ é calculada e comparada a um conjunto de limiares $A_{l 1}$ e $A_{l 2}$ fornecidos a priori. Essa avaliação é realizada da seguinte maneira:

- É procurado um segmento $i_{1} \in I$, tal que sua área $A_{i 1}$ seja $A_{i 1}>A_{l 1}$.

- São procurados dois segmentos $i_{1}, i_{2} \in I$, tal que suas áreas $A_{i 1}$ e $A_{i 2}$ sejam, respectivamente, $A_{i 1}>A_{l 2}$ e $A_{i 2}>A_{l 2}$.

Se qualquer uma das duas condições listadas anteriormente forem atendidas, então as pregas inframamárias foram identificadas e a etapa de refinamento adaptativo é encerrada. Caso contrário, a etapa de refinamento adaptativo é repetida a partir da limiarização. No entanto, o valor de $L$ é decrementado em uma unidade $(L=253)$. A ideia é repetir iterativamente esse procedimento até que a região das pregas inframamárias seja identificada.

Logo, a redução iterativa desse limiar acaba por maximizar a área pertencente às pregas inframamárias, minimizando o efeito causado pela introdução de artefatos indesejados.

Dois limiares associados às áreas são necessários devido a uma avaliação empírica observada durante a criação do método. Em uma série de imagens de teste consideradas, observou-se que a região de alta temperatura associada às pregas inframamárias é composta por uma única componente conexa. No entanto, para um conjunto de imagens, notou-se que a região das pregas inframamárias é composta por duas componentes conexas de menor tamanho. Dessa forma, com a introdução das duas condições mencionadas, foram obtidas às regiões de prega inframamária para um número elevado de imagens advindas das bases de dados utilizadas na literatura.

Uma vez obtida a região das pregas inframamárias, a etapa a seguir, denominada ajuste de curvas, tem por intuito representar de maneira mais fidedigna às curvas que compõem as mamas. Essa representação é feita a partir da modelagem realizada por um polinômio cúbico, que interpola todos os pontos da região inferior das pregas obtidas na etapa anterior.

Finalmente, é utilizado um operador de filtro laplaciano na imagem térmica original, após a conversão de suas cores para tons de cinza. A combinação dos segmentos de ambas as imagens determina a detecção final da mama.

\section{Desenvolvimento}

\subsection{Metodologia}

A metodologia apresentada nesse trabalho foi desenvolvida utilizando o Python 2.7.8 e a biblioteca OpenCV 3.1. Para a geração de resultados e análise de tempos computacionais, foi utilizado um notebook Asus N46V com processador Intel I7, 8 GB de RAM e sistema operacional Windows 10. 
Para a análise de resultados é empregada uma base de dados pública conhecida [Silva et al. 2014], totalizando 283 imagens térmicas. As imagens possuem resolução espacial de 320 x 240 pixels, além de 256 níveis de resolução tonal. Maiores informações sobre a captura das imagens utilizadas podem ser vistas no mesmo trabalho. Alguns exemplos de imagens da base podem ser vistos na Figura 4.
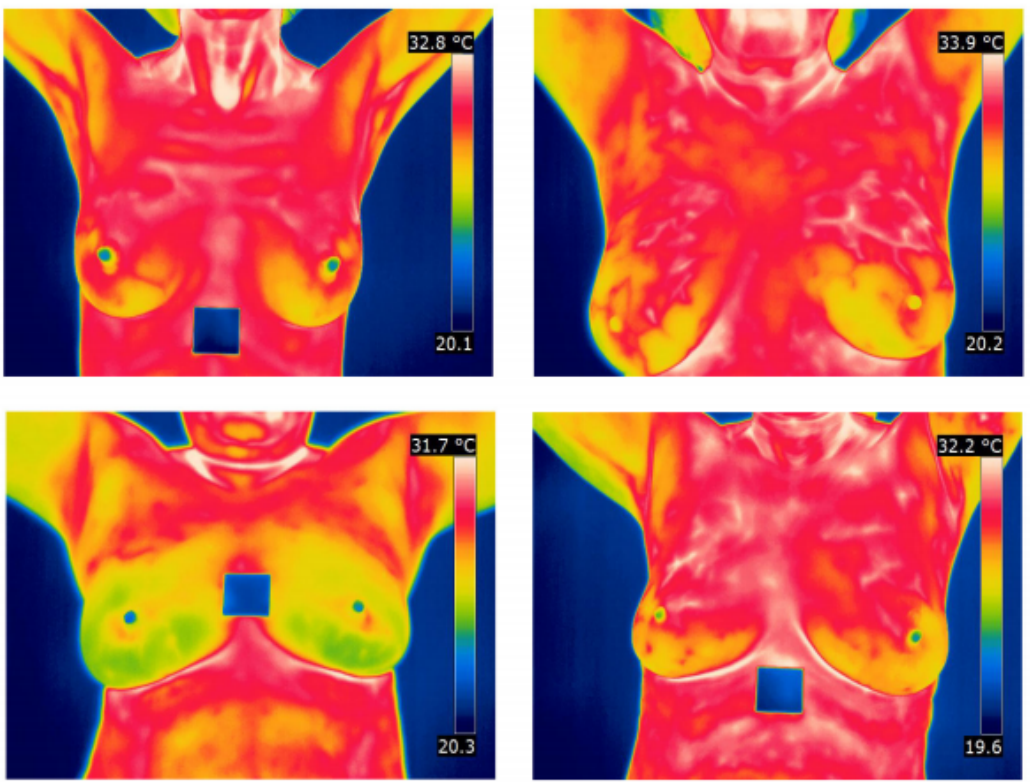

Figura 4. Algumas imagens advindas da base de dados apresentada em [Silva et al. 2014].

Para cada imagem de teste disponível na base de dados, é gerada sua versão ground truth. Essas imagens são construídas a partir de um método de política de votos apresentado em Li et al. [Li et al. 2011], que utiliza diferentes imagens ground truths obtidas manualmente. Essas imagens são comparadas com as obtidas pelo método proposto nesse artigo.

Para avaliar a eficiência desse método, diferentes métricas estatísticas serão consideradas: acurácia, sensibilidade, especificidade, preditividade positiva e negativa. Tais medidas são definidas pelas equações:

$$
\begin{gathered}
\text { Acurácia }(A C C)=\frac{V P+V N}{V P+V N+F P+F N} \\
\text { Sensibilidade }(S E N)=\frac{V P}{V P+F N} \\
\text { Especificidade }(E S P)=\frac{V N}{V N+F P} \\
\text { PreditividadePositiva }(P D P)=\frac{V P}{V P+F P} \\
\text { PreditividadeNegativa }(P D N)=\frac{V N}{V N+F N},
\end{gathered}
$$


em que $V P$ representa as amostras de verdadeiros positivos (pixel que for identificado como parte da região da mama, tanto na imagem teste quanto em sua versão ground truth), $V N$ representa os verdadeiros negativos considerados (pixel que for identificado como parte da região do fundo, tanto na imagem teste quanto em sua versão ground truth), FP denota os falsos positivos avaliados (pixel identificado incorretamente como parte da mama na imagem teste) e, finalmente, $F N$ assinala as amostras de falsos negativos obtidas (pixel erradamente reconhecido como parte do fundo).

Para maximizar os resultados obtidos, foram introduzidos três parâmetros avaliados experimentalmente e empiricamente. A utilização desses parâmetros é necessária pois foi constatado, durante a análise do método, que as pregas inframamárias identificadas podem variar significativamente, dependendo das mamas consideradas. Esses parâmetros estão diretamente relacionados com o procedimento de limiarização com refinamento adaptativo.

O primeiro parâmetro verifica se existe uma única componente conexa que compõe a região das pregas inframamárias, sendo denominado $l a_{1}$. Esse valor determina um limiar mínimo de área medida em pixels que a prega inframamária deve ter, antes da etapa de ajustes das mamas.

Analogamente, o segundo parâmetro analisa se existem duas componentes conexas que definem as pregas inframamárias, sendo esse chamado $l a_{2}$. Essa informação determina uma área mínima a ser medida em pixels para as duas componentes distintas, uma para cada mama.

Finalmente, o último parâmetro se baseia no conceito de que a prega inframamária se localiza nos quadrantes inferiores da imagem térmica. Ele é denominado $l a_{r}$ Dessa maneira, após a etapa de limiarização, todos os pixels $I(x, y)$, tal que

$$
I(x, y)= \begin{cases}0, & \text { se } x, y \geq 0 \text { e } x, y \leq l a_{r}, \\ 1, & \text { se } x, y>l a_{r},\end{cases}
$$

assumindo que $I(x, y)=0$ define um pixel de cor preta na posição $x, y$, e $I(x, y)=1$ torna o pixel daquela posição na cor branca.

No presente trabalho foram realizados diversos experimentos avaliando empiricamente cada parâmetro apresentado. Para os parâmetros apresentados, os seguintes intervalos foram considerados: $l a_{1}=\{1000,1100,1200,1300,1400, \ldots, 2500,2600\}, l a_{2}=$ $\{500,600,700,800,900, \ldots, 1500,1600\}, l a_{r}=\{80,90,100,110,120, \ldots, 140,150\}$. Isso foi feito para que fosse possível determinar qual o conjunto de parâmetros que maximiza as métricas estatísticas apresentadas.

Após computar o método proposto realizando a combinação de todos os valores possíveis dos parâmetros selecionados, foi observado que o conjunto de parâmetros apresentado é similar ao apresentado na Tabela 1.

\subsection{Resultados e Discussão}

A partir do conjunto de parâmetros da Tabela 1 e do método proposto, o presente trabalho será comparado com outros trabalhos importantes apresentados na literatura.

Na Tabela 2, são apresentados os resultados médios de métodos de segmentação automática das mamas, considerando as taxas estatísticas apresentadas em todas as ima- 
Tabela 1. Conjunto de parâmetros que maximiza o resultado das métricas estatísticas consideradas.

\begin{tabular}{ll}
\hline Parâmetro & Valor \\
\hline$l a_{1}$ & 2200 \\
\hline$l a_{2}$ & 150 \\
\hline$l a_{r}$ & 130 \\
\hline
\end{tabular}

gens disponibilizadas na base de dados. Observando os resultados obtidos, é possível concluir que o método apresentado obteve taxas médias altas para todas as medidas estatísticas analisadas. Alguns resultados dos trabalhos referidos são apresentados nas Figuras 5 e 6 .

Tabela 2. Resultados normalizados obtidos pelos trabalhos apresentados na literatura e o método proposto.

\begin{tabular}{llllll}
\hline Trabalhos Considerados & ACC & SEN & ESP & PDP & PDN \\
\hline [Motta 2010] & 0.96 & 0.88 & 0.99 & 0.96 & 0.96 \\
\hline [Marques 2012] & 0.97 & 0.97 & 0.97 & 0.97 & 0.98 \\
\hline Método Proposto & $\mathbf{0 . 9 6}$ & $\mathbf{0 . 9 8}$ & $\mathbf{0 . 9 5}$ & $\mathbf{0 . 9 5}$ & $\mathbf{0 . 9 8}$ \\
\hline
\end{tabular}

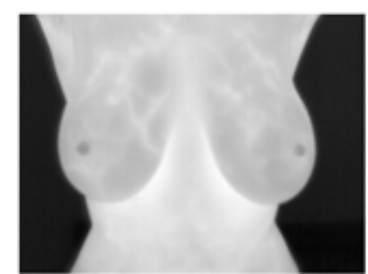

a)

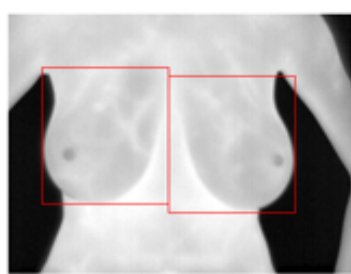

b)

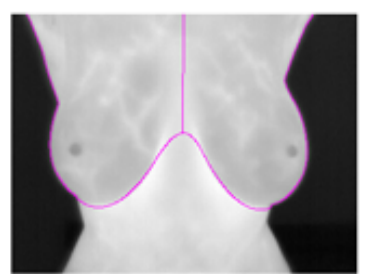

c)

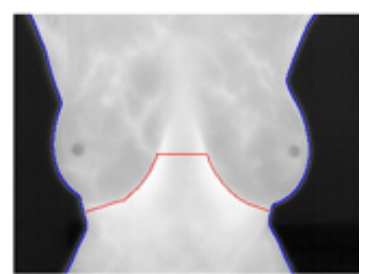

d)

Figura 5. Resultados obtidos para a imagem IR_0100: a) Imagem original de entrada; b) Resultado obtido por [Motta 2010]; c) Resultado obtido por [Marques 2012]; d) Resultado adquirido pelo método proposto nesse trabalho.

Dos três trabalhos considerados, o método apresentado possui as maiores taxas médias de sensibilidade. Isso significa que, dos trabalhos avaliados, a solução proposta possui maior capacidade de identificação de amostras positivas (pertencentes a região de interesse). Essa diferença é superior ao trabalho de [Motta 2010] em uma margem de $10 \%$.

A diferença de acurácia entre [Marques 2012] e o método proposto é de aproximadamente $0,5 \%$, devido a introdução de amostras de falsos positivos na ROI. No entanto, nosso método é bem mais simples de ser implementado, possuindo menos etapas que os trabalhos anteriores. Essa simplicidade sem acarretar em discrepâncias nas métricas estatísticas torna o método muito competitivo e vantajoso para utilização na literatura e na sua utilização em diversas arquiteturas computacionais.

O método de [Marques 2012] possui tempos computacionais de execução muito similares ao trabalho proposto. Isso é visível na Tabela 3, em que os tempos médios em segundos de todos os métodos são apresentados. Se comparado a [Motta 2010], a 


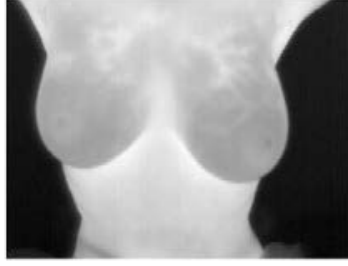

a)

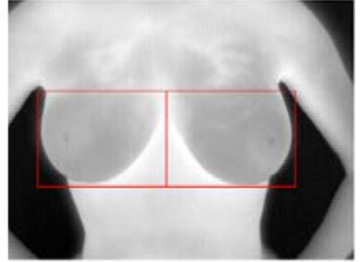

b)

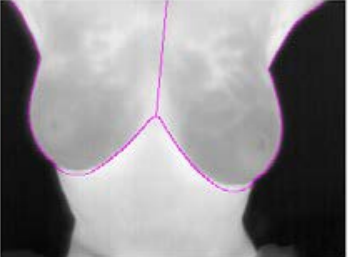

c)

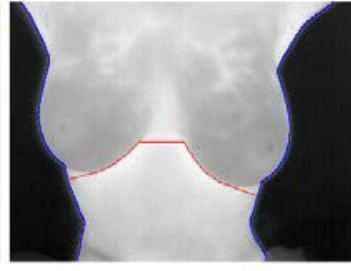

d)

Figura 6. Resultados obtidos para a imagem IR 5986: a) Imagem original de entrada; b) Resultado obtido por [Motta 2010]; c) Resultado obtido por [Marques 2012]; d) Resultado adquirido pelo método proposto nesse trabalho.

diferença torna-se muito mais significativa, sendo aproximadamente 18 vezes mais lento do que o método apresentado nesse trabalho.

Tabela 3. Comparativo de tempo de execução entre os métodos comparados e o trabalho proposto.

\begin{tabular}{ll}
\hline Trabalhos Considerados & Tempo de Execução Médio (s) \\
\hline [Motta 2010] & 36,63 \\
\hline [Marques 2012] & 1,55 \\
\hline Método Proposto & $\mathbf{1 , 9 4}$
\end{tabular}

Para algumas imagens da base de dados, o método não apresenta resultados de maior qualidade. Isso é visível, por exemplo, quando as pacientes possuem pouco volume nas mamas. Isso torna a identificação da região das pregas inframamárias mais complexa. No entanto, como é visível na Figura 7, o impacto desse problema é menos significativo do que em alguns resultados de [Marques 2012] devido a abordagem de refinamento adaptativo, que iterativamente busca uma região de prega inframamária com maior contraste de temperatura, de acordo com a imagem de teste considerada.

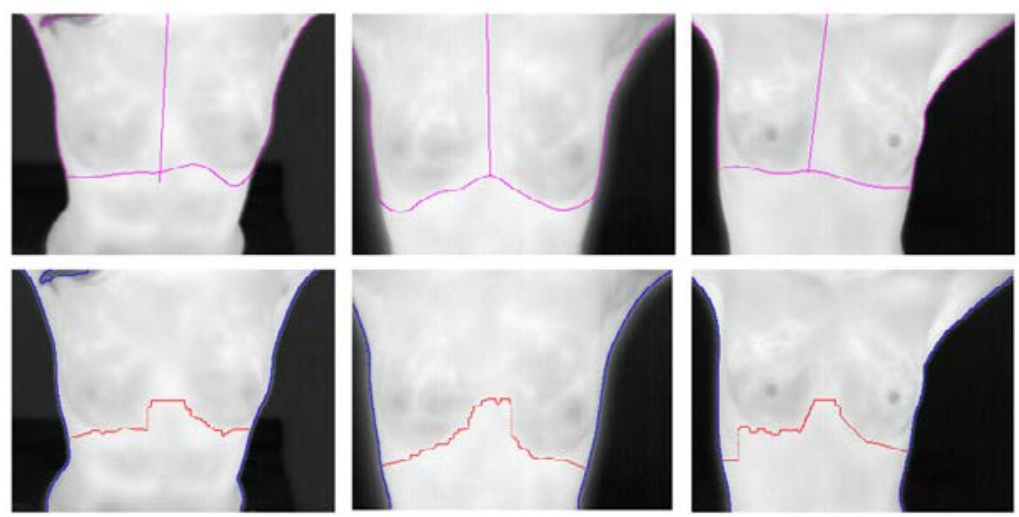

a)

b)

Figura 7. Imagens em que as pacientes possuem mamas de menor volume (respectivamente: IR_5451, IR_7446 e IR_5506): a) Resultados obtidos em [Marques 2012]; b) Resultados obtidos pelo método proposto nesse trabalho.

Outros problemas comuns são: a identificação inapropriada de uma dobra abdominal como parte da região das pregas inframamárias (Figura 8) e a assimetria en- 
tre as mamas (Figura 9). No primeiro caso, a identificação de uma dobra do abdômen como parte das pregas inframamárias introduz uma grande quantidade de falsos positivos, que poderiam ser corrigidos se o ponto de interseção entre os seios fosse melhor assinalado. Com relação à assimetria, o resultado é superior a alguns exemplos apresentados em [Marques 2012], devido a premissa de se assumir que as pregas inframamárias localizam-se nos quadrantes inferiores da imagem e pelo fato de se extrair uma região de prega inframamária de maior densidade de pixels. No entanto, erros ainda ocorrem com certa frequência. É possível corrigí-los adicionando restrições que impeçam que a distância entre as regiões inferiores da mama seja tão contrastante.
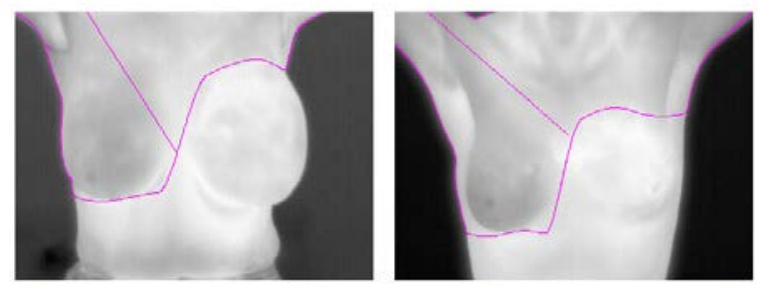

a)
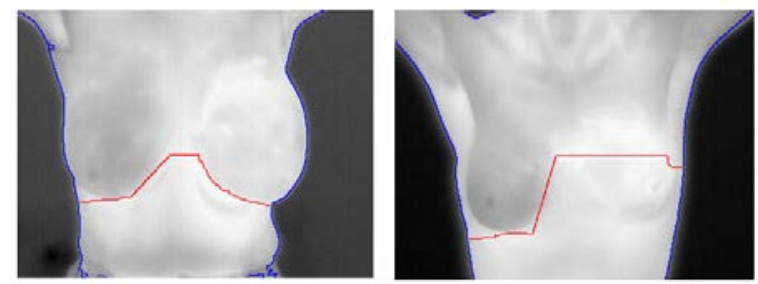

b)

Figura 8. Imagens em que as pacientes possuem dobras abdominais passíveis de confusão com as pregas inframamárias (respectivamente: IR_0889 e IR_1330): a) Resultados obtidos em [Marques 2012]; b) Resultados obtidos pelo método proposto nesse trabalho.
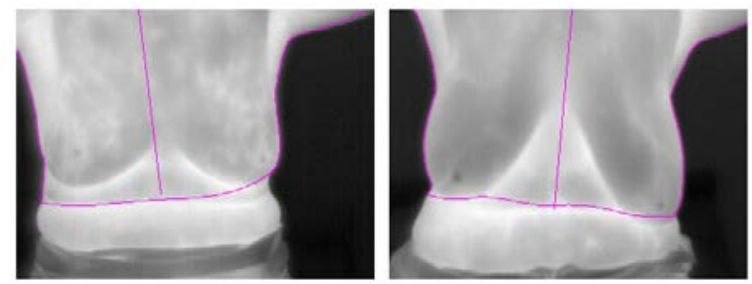

a)
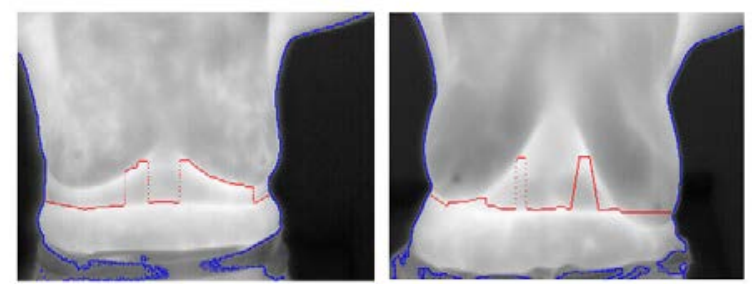

b)

Figura 9. Imagens em que as pacientes possuem mamas com significativa assimetria (respectivamente: IR_8304 e IR_3786): a) Resultados obtidos em [Marques 2012]; b) Resultados obtidos pelo método proposto nesse trabalho.

\section{Considerações Finais}

A detecção precoce do câncer de mama é essencial para melhorar os prognósticos de cura da paciente. Sistemas CAD baseados na simetria da distribuição térmica das mamas se 
mostram eficazes, baratos e pouco invasivos, podendo se tornar relevantes no diagnóstico de doenças da mama. No entanto, para que isso seja feito, torna-se necessário extrair a região de interesse que será avaliado por sistemas de diagnosticação de doenças.

A partir dos resultados apresentados, é visível que o método apresentado torna-se bastante competitivo para uso em situações reais, devido a acurácia do resultado final, bem como o tempo de execução médio que é relativamente baixo. Além disso, a metodologia é fácil de ser desenvolvida computacionalmente, pois possui muito menos etapas que os outros trabalhos da literatura e requer poucos recursos computacionais (tanto de hardware quanto de software) para o seu funcionamento.

Para trabalhos futuros, espera-se:

- Adaptar o método para imagens com câmeras em ângulos distintos;

- Utilizar técnicas para extração de atributos e características da mama para diagnóstico de doenças. Esses atributos podem ser geométricos ou de textura;

- Desenvolver sistemas CAD para diagnóstico de doenças diversas da mama a partir do algoritmo de segmentação proposto.

\section{Referências}

Arora, N. M., Martins, D., Ruggerio, D., Tousimis, E., Swistel, A. J., Osborne, M. P., and Simmons, R. M. (2008). Effectiveness of a noninvasive digital infrared thermal imaging systems in the detection of breast cancer. American Journal of Surgery, 196(4):523-526.

Gore, J. P. and Xu, L. X. (2014). Thermal Imaging for Biological and Medical Diagnostics. CRC Press.

HCB (2015). Informação: Saiba quais são os tipos de câncer mais comuns no brasil. http://www.hcancerbarretos.com.br/82-institucional/ noticias-institucional/1300-informacao-saiba-quais-saoos-tipos-de-cancer-mais-comuns-no-brasil. Acesso em: 29 de outubro de 2015.

INCA (2015). Tipos de câncer. http://www2.inca.gov.br/wps/wcm/ connect/tiposdecancer/site/home/mama. Acesso em: 07 de novembro de 2015.

Li, X., Aldridge, B., Fisher, R. B., and Rees, J. (2011). Estimating the ground truth from multiple individual segmentations incorporating prior pattern analysis with application to skin lesion segmentation. In ISBI, pages 1438-1441. IEEE.

Marques, R. S. (2012). Segmentação automática das mamas em imagens térmicas. Master's thesis, Instituto de Computação, Universidade Federal Fluminense, Niterói, RJ, Brasil.

Motta, L. S. (2010). Obtenção automática da região de interesse em termogramas frontais da mama para o auxílio à detecção precoce de doenças. Master's thesis, Instituto de Computação, Universidade Federal Fluminense, Niterói, RJ, Brasil.

Silva, L. F., Saade, D. C. M., Sequeiros-Oliveira, G. O., Silva, A. C., Bravo, R. S., and Conci, A. (2014). A new database for breast research with infrared image. Journal of Medical Imaging and Health Informatics, 4(1):92-100. 